\title{
The Adnexal Mass: Benign or Malignant? Evaluation of Risk of Malignancy Index
}

\author{
Shamsun Nahar ${ }^{1}$ Latifa Shamsuddin ${ }^{2}$ \\ ${ }^{1}$ Associate Professor \& Head, Dept. of OB-GYN, Central Medical College, Comilla. ${ }^{2}$ Professor \& Ex-Chairman, Dept. of OB-GYN, Bangabandhu \\ Sheikh Mujib Medical University ( BSMMU), Dhaka.
}

\begin{abstract}
:
Background: Screening programme for ovarian malignancy is an international public demand. Ojective: To validate a risk of malignancy index (RMI) incorporating serum CA125, abdominal ultrasound findings and menopausal status for preoperative diagnosis of ovarian malignancy among patient with adnexal mass. Methods: This prospective observational study was conducted on 57 patients having adnexal mass admitted consecutively for surgical exploration in BSMMU from January 2000 to March 2001. S. CA125 assay \& abdominal ultrasonography was done within 10 days preoperatively. Ultrasound score $(0,1 \& 3)$ using 5 sonographic features \& menopausal score $(1 \& 3)$ were calculated. RMI was detected by the formula "RMI = Ultrasound score $\otimes$ Menopausal score $\otimes \mathrm{S}$. CA125 level". Definitive diagnosis was based on histopathological examination. Sensitivity, specificity, positive and negative predictive values of S.CA 125 , ultrasound score \& menopausal score individually and in the form of RMI to diagnose Ovarian malignancy preoperatively were detected . For statistical analysis Chi square \& student's 't' test were used. Results: Sensitivity, specificity, positive and negative predictive values of RMI at the cut-off value of 200 were $78 \%, 88 \%, 58 \%$ and $95 \%$ respectively. Conclusion: RMI was more accurate than any individual criterion in diagnosing Ovarian malignancy. This scoring system can be introduced easily into clinical practice to facilitate selection of patient for appropiate surgery at proper centre by skilled surgeon .
\end{abstract}

Key words: RMI , S.CA125, Ultrasound findings, Menopausal status \& Adnexal mass .

[BSMMUJ $2013 ; 6$ (2) : 127-134]

\section{Introduction:}

The management of a woman having adnexal mass is a problem frequently faced by the Gynecologists. It may represent a wide and varied differential diagnosis including both benign and malignant gynecological conditions and also some non-gynecological conditions. ${ }^{1}$ In a clinically diagnosed adnexal mass traditional strategy for establishing final diagnosis has been to perform an exploratory laparotomy. ${ }^{2}$

Ovarian malignancy is an important adnexal pathology. It shows the highest mortality among the gynecological malignancy and has become the greatest challenge in

Address for Correspondence: Dr. Shamsun Nahar Associate Professor, Dept. of OB-GYN, Central Medical College, Paduar Bazar, Bishwa Road Comilla, Bangladesh. Mobile: 01716551941,E-mail: drsn101@gmail.com gynecological oncology throughout the globe including Bangladesh.

In recent years, there has been developed considerable international public demand for ovarian malignancy screening. Multiple studies have concluded the in-effectiveness of the screening programme due to the absence of pre-cancerous condition and low prevalence of ovarian malignancy among the population.$^{3}$

The prognosis of ovarian malignancy may be greatly influenced by appropriate first line surgery. The initial laparotomy is important for accurate staging and cytoreductive surgery. ${ }^{4}$ Staging of neoplastic disease has assumed great importance in selection of the best form of therapy for the patient. ${ }^{5}$ The aim of cytoreductive surgery is to achieve optimal residue and the amount of residual 
disease after primary surgery is the most important prognostic factor ${ }^{6,7}$ Moreover, chemotherapy and radiotherapy are likely to be effective in optimal residual disease.

However, in-spite of known benefit of meticulous comprehensive surgical staging and cytoreductive surgery, many women do not receive appropriate first line surgery. This is because of difficulty in making an accurate pre-operative diagnosis of ovarian malignancy as well as skilled and lengthy surgery of cytoreduction. In practice, the diagnosis of ovarian malignancy is often difficult to make pre-operatively and inappropriate \& inadequate surgical exploration by junior or inexperienced surgeon at improper centre is not uncommon . Therefore, sensitive and specific methods for diagnosis of ovarian malignancy are required which may influence the selection of the institution and also the seniority and expertise of surgeon. This would provide a rational basis for referral before diagnostic laparotomy. ${ }^{89}$

Ultrasound reflects the gross morphological characteristics of tumour, not the histology. Evidence of solidity , mixed echogenicity, multiloculation, thick/irregular wall and septations, papillary projections, bilaterality, larger size, ascites \& evidence of metastasis are suggestive of malignancy. ${ }^{10,11}$ To improve ultrasonic diagnostic accuracy in ovarian mass, many scoring systems have been developed for objective evaluation of changes in many morphological parameters.$^{12}$

The tumour marker, CA125 mostly elevates in epithelial ovarian malignancy. Its normal value is up to $35 \mathrm{U} / \mathrm{ml}$. The diagnostic accuracy of CA125 assay is sufficiently high to be of clinical value to discriminate benign from malignant ovarian conditions. ${ }^{13,14}$ S.CA125 also rises in other pelvic malignancies and various benign conditions. It may or may not rise in non-epithelial ovarian malignacy. ${ }^{15}$

The risk of malignancy index (RMI) is a simple but novel scoring system introduced by Jacobs \& his associates in 1990 utilizing 3 diagnostic criteria - ultrasound findings , serum CA 125 level and menopausal status of the patient . These researchers formulated as, $\mathrm{RMI}=\mathrm{U} \times \mathrm{M} \times \mathrm{S}$. $\mathrm{CA} 125$ ( $\mathrm{U}=$ ultrasound score, $\mathrm{M}=$ menopausal score and
S. CA125 = absolute value of serum CA125). They suggested that to distinguish benign and malignant adnexal mass the RMI is superior to 3 findings in isolation and a clinician would expect to classify correctly approximately $90 \%$ of patient with benign or malignant adnexal mass using the RMI . RMI utilizes currently available non-invasive tests, which can be applicable in clinical practice and would provide rational basis for specialist referral of patient with malignancy prior to surgery.$^{16}$

This study was conducted to validate the role of RMI to discriminate malignant from benign ovarian conditions in our population .

\section{Methods:}

This was a prospective observational study. The study was conducted during the period of January 2000 to March 2001 in the Department of Obstetrics and Gynecology of BSMMU, Dhaka, with the full co-operation of Department of Clinical Pathology, Department of Pathology \& Department of Radiology \& Imaging of BSMMU, Dhaka. The girls and women admitted consecutively in the Department of Obstetrics and Gynecology for the surgical exploration of adnexal mass and having informed willingness in participation into the study $(\mathrm{n}=57)$ were enrolled for the study. Patient with histo-pathological report showing non-adnexal mass was excluded .

Proper permission to conduct the study was taken from the chairmen of the concerned Departments after approval of the protocol . Verbal consent from each patient was obtained after informing about the nature and aim of the study .

Data was collected in data collection instrument which was a pre-designed data sheet of mixed form and questionnaire type. 2 methods were followed for data collection - by interview and by some investigations . Measurement of outcome variables were serum CA125, ultrasound score, menopausal score and RMI .

In interview method, the socio-demographic characteristics and clinical informations were recorded by interview. Age was recorded in nearest full years as stated by the patient. Socio-economic status was categorized on monthly income as poor ( $<3000$ Taka), middle (30006000 Taka) and rich (>6000 Taka). 
In investigative method, $2 \mathrm{ml}$ of peripheral venous blood sample was obtained with full aseptic precaution by disposable syringe from each patient within 10 days preoperatively. Blood was drawn for the single time from each patient and was poured into a clean glass test tube. Then the sample was allowed to clot at room temperature and the serum was separated. S.CA 125 was determined by the technician using commercial kit (IMX, Abbott laboratories , USA) by Micropartical Enzyme Immuno Assay ( MEIA) technique at the Department of clinical pathology, BSMMU, Dhaka.

Ultrasound scan of whole abdomen was performed by the Sonologist of Radiology and Imaging Department using 3.5 $\mathrm{MHz}$ transabdominal transducer in full bladder technique within 10 days preoperatively. Ultrasonic features noted were solidity, multilocularity, bilaterality , ascites and evidence of intra-abdominal metastasis . Ultrasound score (U) was calculated for each patient as follows - score of 0 , if none of the features was present, score of 1 if one of the features was present and score of 3 , if $\geq 2$ of the features were present .

Post menopausal status was defined as $\geq 1$ year of amenorrhoea or $\geq 50$ years of age, if previously had undergone hysterectomy. Pre-menopausal status was considered as the state outside the aforementioned criteria. Menopausal score (M) was scored as 1 if pre-menopausal and 3 if postmenopausal.

RMI score was calculated using the formula proposed by Jacobs et al. (1990) : RMI $=$ U X M X serum CA125 where, $\mathrm{U} \& \mathrm{M}$ were ultrasound and menopausal score as defined above .

Immediately after surgery the resected specimen was sent to the Department of Pathology preserving in formalin solution. Histopathological examination was performed by the Pathologist . Definitive diagnosis was based on histopathological finding. In one case of illegal ectopic pregnancy, the surgical diagnosis was assumed to be correct as histopathological examination was not performed on patient's request.
Data was analyzed using SPSS program of computer with the help of statistician. SD was taken as a measure of variation and frequency of data was expressed as mean \pm SD. Student's ' $t$ ' test was used to test the differences in distribution of age , parity, serum CA125, ultrasound score and RMI among the patients with benign and malignant adnexal mass. Chi-square test was applied in testing differences in distribution of socio-economic and menopausal status in the same groups . Level of significance was expressed as $p$ value and values showing $<.05$ was considered as significant to assess the performance of serum CA125, ultrasound score , menopausal score and RMI score to discriminate malignant from benign adnexal mass. The sensitivity, specificity, positive predictive values(PPV) and negative predictive values (NPV) were calculated at different cut-off values.

\section{Results:}

Though 57 patients were initially enrolled for the study, 3 were dropped out due to refusal of surgery by patient herself in 1 and decision of giving primary chemotherapy in the rest 2. Later on another 5 were excluded from the study where 4 were proved to be non-adnexal malignancy (uterine leiomyosarcoma - 2, fibrosarcoma - 1, and malignant fibrous histocytoma - 1) and 1 to be uterine leiomyoma on histopathological examination .Thus, 49 patients comprised the study of population .

Out of 49 patients , $9(18 \%)$ had ovarian malignancy , where majority(3) were serous cystadeno-carcinoma. On the other hand , $40(82 \%)$ benign adnexal mass were detected. Majority (9) were functional cyst followed by serous cystadenoma (7) , ovarian endometrioma(6), dermoid cyst (5) and the rest were other benign pelvic conditions.

Data regarding the patient characteristics and relevant informations have been presented in the following different Tables and Figures. 

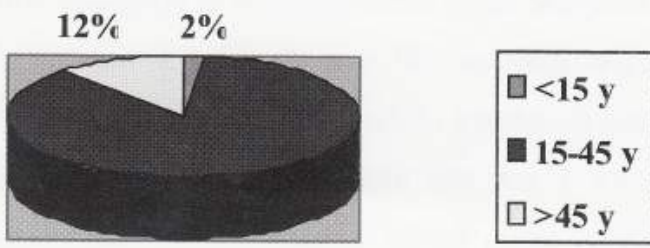

$86 \%$

Fig-1: Age distribution of the study patients $(n=49)$

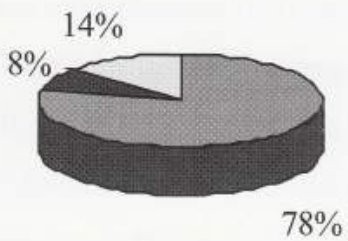

口House wife

Working

口Student

Fig-2: Occupation distribution of the study patients ( $\mathrm{n}=$ 49)
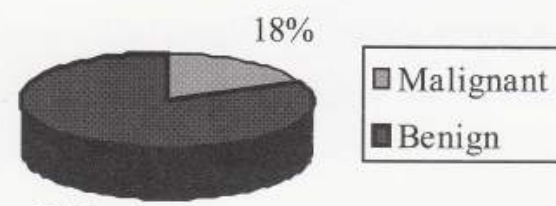

abenign

$82 \%$

Fig-3: Distribution of Benign and Malignant Adnexal Mass in Study Patients ( $\mathrm{n}=49$ )

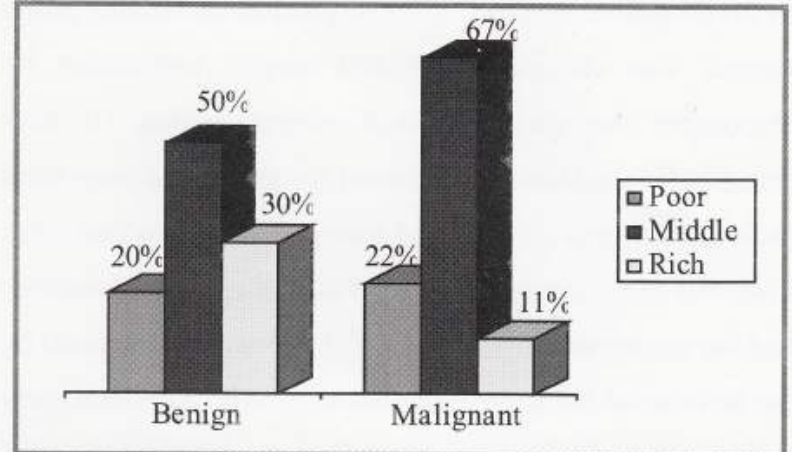

Fig-4: Distribution of Socio-economic status in Patients with Benign and Malignant Adnexal Mass

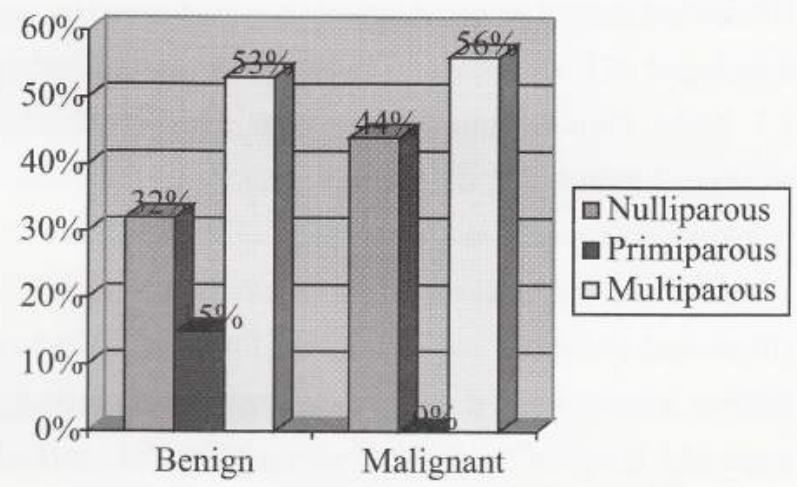

Fig-5: Distribution of Parity in Patients with Benign and Malignant Adnexal Mass

Table-I

Distribution of Serum CA 125 levels in Patient with Adnexal Mass (n-49)

\begin{tabular}{llll}
\hline \multicolumn{1}{c}{ Diagnosis } & \multicolumn{1}{c}{ Number } & \multicolumn{1}{c}{$<5 \mathrm{U} / \mathrm{ml}$} & $>35 \mathrm{U} / \mathrm{ml}$ \\
\hline Benign Adnexal Mass & $40(82 \%)$ & $27(68 \%)$ & $13(32 \%)$ \\
Functional cyst & 9 & 8 & 1 \\
Serous cystadenoma & 7 & 6 & 1 \\
Mucinous cystadenoma & 2 & 2 & 0 \\
Dermoid cyst & 5 & 3 & 2 \\
Parovarian cyst & 3 & 3 & 0 \\
Ovarian endometrioma & 6 & 3 & 3 \\
Inflammatory T -O mass & 2 & 0 & 1 \\
Tubercular pseudoc yst & 3 & 0 & 3 \\
Residual ovarian syndrome & 2 & 1 & 1 \\
Tubal pregnancy & 2 & 1 & 1 \\
Malignant Adnexal Mass & $9(18 \%)$ & $1(11 \%)$ & $8(89 \%)$ \\
Serous cystadeno -Ca & 3 & 0 & 3 \\
Mucinous cystadeno -Ca & 1 & 0 & 1 \\
Endometroid adeno Ca & 1 & 0 & 1 \\
Granulosa cell tumour & 1 & 0 & 1 \\
Dysgerminoma & 1 & 1 & 0 \\
Endodermal sinus tumour & 1 & 0 & 1 \\
Mixed Germ cell tumour & 1 & 0 & 1 \\
\hline
\end{tabular}


Table-II

Distribution of Age, Parity, Socio-economic status among Patients with Adnexal Mass (n-49)

\begin{tabular}{|c|c|c|c|c|c|}
\hline Parameters & & $\begin{array}{l}\text { Benign } \\
(\mathrm{n}=40)\end{array}$ & $\begin{array}{c}\text { Malignant } \\
(\mathrm{n}=9)\end{array}$ & P Value & $\begin{array}{c}\text { Statistical } \\
\text { Tests }\end{array}$ \\
\hline \multicolumn{6}{|l|}{ Age (years) : } \\
\hline Range & & $17-80$ & $11-60$ & & Unpaired \\
\hline Mean \pm SD & & $33.42 \pm 12.63$ & $33.11 \pm 16.51$ & $0.950^{\mathrm{NS}}$ & Student's \\
\hline Median & & 30 & 30 & & ' $\mathrm{t}$ ' test \\
\hline \multicolumn{6}{|l|}{ Parity : } \\
\hline Range & & $0-10$ & $0-9$ & & Unpaired \\
\hline Mean \pm SD & & $2.20 \pm 2.49$ & $2.67 \pm 3.12$ & $0.630 \mathrm{NS}$ & Student's \\
\hline Median & & 2 & 2 & & 't' te st \\
\hline \multicolumn{6}{|l|}{ Socio -economic status } \\
\hline Poor & & $8(20 \%)$ & $2(22 \%)$ & & \\
\hline Middle & & $20(50 \%)$ & $6(67 \%)$ & & \\
\hline Rich & & $12(30 \%)$ & $1911 \%)$ & 0.499 NS & Chi -square test \\
\hline
\end{tabular}

a: values were expressed in frequency

NS: not significant

Table-III

Distribution of S.CA125 Level, Ultrasound score, Menopausal status and RMI among Patients with Adnexal Mass (n-49)

\begin{tabular}{|c|c|c|c|c|c|}
\hline Parameters & & $\begin{array}{l}\text { Benign } \\
(\mathrm{n}=40)\end{array}$ & $\begin{array}{c}\text { Malignant } \\
\quad(n=9)\end{array}$ & P Value & $\begin{array}{c}\text { Statistical } \\
\text { Tests }\end{array}$ \\
\hline \multicolumn{6}{|l|}{ S. CA $125(\mathrm{U} / \mathrm{ml})$ : } \\
\hline Range & & $1.96-600$ & $9.47-600$ & & Unpaired \\
\hline Mean \pm SD & & $63.01 \pm 116.80$ & $292.59 \pm 249.46$ & $.000^{* * *}$ & Student's \\
\hline Median & & 21.2 & 214.64 & & ' $\mathrm{t}$ ' test \\
\hline \multicolumn{6}{|l|}{ Ultrasound Score: } \\
\hline Range & & $0-3$ & $1-3$ & & Unpaired \\
\hline Mean \pm SD & & $1.10 \pm 1.13$ & $2.56 \pm .88$ & $.001 * * *$ & Student's \\
\hline Median & & 1 & & & t'test \\
\hline \multicolumn{6}{|l|}{ Menopausal status } \\
\hline Premenopausal & & $36(90 \%)$ & $7(78 \%)$ & & Chi -square \\
\hline Postmenopausal & & $4(10 \%)$ & $2(22 \%)$ & $.312^{\mathrm{NS}}$ & test \\
\hline \multicolumn{6}{|l|}{ RMI : } \\
\hline Range & & $0-1800$ & $9.47-5400$ & & Unpaired \\
\hline Mean \pm SD & & $121.31 \pm 328.45$ & $1667.30 \pm 2185.87$ & $0.000 * * *$ & Student's \\
\hline Median & & 12.22 & 643.92 & & $\mathrm{t}$ test \\
\hline
\end{tabular}

a : Values were expressed in frequency

*** Significant at $\mathrm{P}<.001 \quad$ NS : Not significant 
Table-IV

Sensitivity, Specificity, PPV \& NPV of S.CA125, Ultrasound score, Menopausal status and RMI in Diagnosis of Malignant Adnexal Mass (n- 49)

\begin{tabular}{|c|c|c|c|c|}
\hline Parameters & $\begin{array}{c}\text { Sensitivity } \\
(\%)\end{array}$ & $\begin{array}{c}\text { Specificity } \\
(\%)\end{array}$ & PPV (\%) & NPV (\%) \\
\hline \multicolumn{5}{|l|}{ S. CA125 (U/ml): } \\
\hline$>30$ & 89 & 58 & 32 & 96 \\
\hline$>50$ & 78 & 70 & 37 & 93 \\
\hline$>70$ & 78 & 78 & 44 & 94 \\
\hline$>90$ & 67 & 85 & 50 & 92 \\
\hline$>120$ & 67 & 88 & 55 & 92 \\
\hline \multicolumn{5}{|l|}{ Ultrasound Score: } \\
\hline$\geq 1$ & 100 & 38 & 26 & 100 \\
\hline 3 & 78 & 80 & 47 & 94 \\
\hline Menopausal Status: & 22 & 90 & 33 & 84 \\
\hline \multicolumn{5}{|l|}{ RMI: } \\
\hline 25 & 89 & 58 & 32 & 96 \\
\hline 50 & 78 & 70 & 37 & 93 \\
\hline 75 & 78 & 83 & 50 & 94 \\
\hline 100 & 78 & 83 & 50 & 94 \\
\hline 150 & 78 & 85 & 54 & 94 \\
\hline 200 & 78 & 88 & 58 & 95 \\
\hline 250 & 78 & 88 & 58 & 95 \\
\hline
\end{tabular}

\section{Discussion :}

Ovarian malignancy is a major cancer killer in gynaecological oncology throughout the globe including Bangladesh. Absence of pre-cancerous condition, silent nature or non-specific symptoms at earlier stages and low prevalence are responsible factors for the ineffectiveness of screening programme . Detection at earlier stages can at least reduce the cancer mortality by giving opportunity of primary cytoreductive surgery with accurate staging. But the diagnosis of this adnexal malignancy is difficult due to existence of wide varieties of adnexal pathologies including both the gynaecological and non-gynaecological. A reliable diagnostic method is very much demanding to reduce the problem. Because only then appropriate surgery by experienced gynaecologist at institution with adequate facilities locally or after referral may be possible.

RMI is reliable diagnostic scoring system to discriminate malignant from benign adnexal mass. To test the validity of RMI this study has been performed. Therefore, the sensitivity, specificity and predictive values of serum CA125, ultrasound score, menopausal status and RMI to discriminate malignant from benign adnexal mass were determined and compared.

In present study, optimal cut-off value of serum CA125 was $>120 \mathrm{U} / \mathrm{ml}$ in contrast to other studies, where the cut-off values ranges from $30-70 \mathrm{U} / \mathrm{ml}$. The specificity 
$(88 \%)$ of serum CA125 was acceptable and comparable to previous studies of Jacobs et al. ${ }^{9}(75 \%)$ and Davies et al. ${ }^{8}$ $(85 \%)$. But somewhat lower sensitivity was found than those researchers ( $67 \%$ vs $81 \%$ and $70 \%$ respectively), which may be due to finding of lower number of malignant adnexal mass in this study ( $18 \%$ vs $29 \%$ in Jacobs et al. $^{9}$ and $30 \%$ in Davies et al. ${ }^{8}$ ).

Both sensitivity and specificity of ultrasound score in present study were in agreement with the previous studies. In comparison to Jacobs et al. ${ }^{9}$ sensitivity was higher(78\% vs $71 \%$ ) and specificity was lower ( $80 \%$ vs $83 \%$ ) but the variations were acceptable. In comparison to Davies et al. ${ }^{8}$ specificity was higher ( $80 \%$ vs $74 \%$ ), but sensitivity was slightly lower ( $78 \%$ vs $87 \%$ ) in our population, that was also acceptable. Moreover, like the previous researchers, it was found that along with malignancy, positive ultrasound finding was also associated with benign mass.

Performance of menopausal status was found to be less sensitive in present study ( $22 \%$ vs $79 \%$ and $59 \%$ in the study of Jacobs et al. ${ }^{9}$ and Davies et al. ${ }^{8}$ respectively) though specificity was higher ( $90 \%$ vs $53 \%$ and $87 \%$ in previous 2 studies respectively). Very low sensitivity may be due to lower number of post-menopausal patients in this study.

In present study, the optimal test performance of RMI to discriminate malignant from benign adnexal mass determined at cut-off value of both 200 and 250 . Sensitivity, specificity, positive and negative predictive values were $78 \%, 88 \%, 58 \%$ \& $95 \%$ respectively . This unexpected finding of same results at both cut-off values were possibly due to contribution of RMI score of 200 and 250 by the same group of patient as sample size was small.

The diagnostic measures of RMI at optimal cut-off value(200 or 250$)$ need to be explained. Sensitivity of $78 \%$ indicates that RMI can detect $78 \%$ of ovarian malignancy when RMI score is 200 or 250 . Specificity of $88 \%$ indicates that RMI can detect $88 \%$ of benign adnexal mass at that score of RMI. On the other hand, positive predictive value of $58 \%$ means that $58 \%$ of adnexal mass will be ovarian malignancy at that score. Negative predictive value of $95 \%$ means that $95 \%$ of adnexal mass will be benign at that score.In this study, RMI sensitivity (78\%) was higher than the sensitivity ( $71 \%$ ) found by Tingulstad et al. ${ }^{2}$, but it was lower than that of Davies et al. ${ }^{8}$ and Jacobs et al. ${ }^{9}$ which was $87 \%$ and $85 \%$ respectively. The specificity was almost similar to Davies et al. ${ }^{8}$,but slightly lower than other two studies. The negative predictive value was higher $(95 \%)$ but positive predictive value was lower $(58 \%)$ than those of other reseachers. This study also showed that RMI could discriminate $83 \%$ of malignant from benign mass. But this discrimination was $91 \%$, $88 \%$ and $84 \%$ in the studies of Jacobs et al. ${ }^{9}$, Davies et $\mathrm{al}^{8}{ }^{8}$, and Tingulstad et $\mathrm{al}^{2}{ }^{2}$ respectively. The overall diagnostic accuracy of RMI was in agreement with the previous studies, but slight variation may be due to smaller sample size.

\section{Conclusion:}

RMI is a simple, non-invasive and reliable diagnostic method, which can be useful in clinical practice . Ultrasonic features used in RMI can be easily identified by sonologist with trans-abdominal transducers and now a days ultrasound is widely available and less expensive in Bangladesh.

Routine use of RMI at institution with proper facilities in cases of ovarian pathology could provide early and reliable prediction of ovarian malignancy with consequent appropriate surgical approach. This includes preoperative consultation with general surgeon, necessary preoperative preparation, planning of extendable midline incision and adequate surgery. Moreover, detection of ovarian malignancy at centre with lack of adequate facilities of appropriate surgical approach may provide a basis for referral to proper institution, to experienced gynaecologist or to the gynaecologist wtih special interest in oncology Such a study on larger population may further improve diagnostic precision of RMI and that is certainly demanding .

\section{Acknowledgements:}

This work was supported by grant from Bangladesh Medical Research Council (BMRC) and also by supplying kits for S.CA125 assay from Abbott Lab. Ltd. Karachi . 


\section{Reference:}

1. Sanfilippo JS and Rock JA. Surgery for Benign Disease of the Ovary. In: Rock JA and Thompson JD(editors). Te Linde's Operative Gynecology. 8th ed . Philadelphia: Lippincott-Raven Publishers, 1997: pp 625-644.

2. Tingulstad S, Hagen B, Skjeldestad FE, Onsrud M, Kiserud T, Halvorsen T, Nustad K. Evaluation of a Risk of Malignancy Index Based on Serum CA125, Ultrasound Findings and Menopausal Status in the preoperative Diagnosis of Pelvic Masses. Br J 0bstet Gynecol 1996; 103:826-831.

3. Bell R, Petticrew M, Sheldon T. The performance of screening Tests for Ovarian Cancer: Results of a Systemic Review. Br J Obstet Gynaecol 1998;105:1136-1147.

4. Gillis CR, Hole DJ, Still RM, Davis J, kaye SB. Medical Audit, Cancer Registration and Survival in Ovarian Cancer. Lancet 1991; 337: 611-612.

5. Cotran RS, Kumar V , Collins T. Robins Pathologic Basis of Disease . 6th ed. Philadelphia: WB Saunders Company, 1999: pp. 260-327.

6. Delgado G, Dram DH and Petrilli EG. Stage III Epithelial Ovarian Cancer: The Role of Maximal surgical Reduction. Gynecol Oncol 1984; 18: 293 .

7. Kehoe S, Powell J, Wilson S, Woodman C. The influence of the operating Surgeon's Specialization on Patient Survival in Ovarian Carcinoma . Int J Cancer 1994; 70:1014-1017.

8. Davies AP, Jacobs I, Woolas R, Fish A, Oram D. The Adnexal Mass: Bengin or Malignant ? Evaluation of a Risk of malignacy index. Br J Obstet Gynaecol 1993; 100:927-931.
9. Jacobs I, Oram D, Fairbanks J, Turner J, Frost C, Grudzinskas JG. A Risk of Malignancy Index incorporating CA125, Ultrasound finding and Menopausal Status for the Accurate Preoperative Diagnosis of Ovarian Cancer. Br. J Obstet Gynaecol 1990; 97: 922-929.

10. Moyle JW, Rochester D, Sider L, Shrock K, Krause P. Sonography of Ovarian Tumours : Predictability of tumour type. AJR $1983 ; 141: 985$.

11. Kobayashi M. Use of Diagnostic Ultrasound in Trophoblastic Neoplasm and Ovarian Tumours. Cancer 1976 ; 38:441.

12. Kurjak A , Pradanijc M .New Scoring System for Prediction of Ovarian Malignancy Based on Transvaginal Color Doppler Sonography . J Ultrasound Med 1992; 11: 632-638.

13. Di-Xia C, Schwartz PE , Xinguo L and Zhan Y. Evaluation of CA125 Levels in Differentiating Malignant from Benign Tumors in Patients with Pelvic Masses. Obstet Gynecol 1988,72:23.

14. Einhorn N, Sjovall K, Knapp RC, Bast RC Jr. Prospective Evaluation of Serum CA 125 levels for early detection of Ovarian Cancer. Obstet Gynecol 1992;80: 14-18.

15. Bast RC Jr, Klug Tl, John E, Jenison E, Niloff JM, Lazarus H, Berkowitz RS et al. A Radioimmunoassay Using Monoclonal Antibody to Monitor the Course of Epithelial Ovarian Cancer. N Engl J Med 1983 ; $309: 883-887$.

16. Morgante G, Marca AI, Ditto A, Leo VD . Comparison of Two Malignancy Risk Indices Based on Serum CA125, Ultrasound Score and Menopausal Status in Diagnosis of Ovarian Masses. Br J Obstet and Gynaecol 1999; $106: 524-527$. 